\title{
Chaotic Features of the Forward Shift Map on the Generalized $m$-Symbol Space
}

\author{
Hena Rani Biswas ${ }^{1, *}$, Md. Shahidul Islam² \\ ${ }^{1}$ Department of Mathematics, University of Barishal, Barishal-8200, Bangladesh. \\ ${ }^{2}$ Department of Mathematics, University of Dhaka, Dhaka-1000, Bangladesh.
}

How to cite this paper: Hena Rani Biswas, Md. Shahidul Islam. (2020) Chaotic Features of the Forward Shift Map on the Generalized $m$-Symbol Space. Journal of Applied Mathematics and Computation, 4(3), 104-112.

DOI:10.26855/jamc.2020.09.006

Received: August 17, 2020

Accepted: September 18, 2020

Published: September 30, 2020

*Corresponding author: Hena Rani Biswas, Department of Mathematics, University of Barishal, Barishal-8200, Bangladesh.

Email:biswas.hena@yahoo.com

\begin{abstract}
In this paper, we study some chaos related properties of the forward shift map $\sigma^{+}$ on the generalized one-sided symbol space $\sum_{m}^{+}, m(\geq 2) \in N$. We consider several notions of chaos available in the contemporary literature. In this paper, we prove that $\sigma^{+}$is Devaney chaotic, Auslander-Yorke's chaotic and generically $\delta$-chaotic. We prove that $\sigma^{+}$is exact Devaney chaotic and as a consequence is mixing Devaney Chaotic and weak mixing Devaney Chaotic. We also provide examples to show that the forward shift map $\sigma^{+}$on $\sum_{m}^{+}$is topologically conjugate to the map on the space or equivalently conjugate to the map $f_{m}(x)=m x(\bmod 1)$ on the space $R / Z$. Finally, we give a counterexample to prove that not all topologically transitive maps are totally transitive. We also give an example of a continuous function that is topologically transitive but not chaotic.
\end{abstract}

\section{Keywords}

Forward Shift Map, Symbol Space, Chaotic Dependence on Initial Conditions, Totally Transitive, Topological Conjugacy

\section{Introduction}

In this paper, we consider the space $\sum_{m}^{+}$which is $m$-symbol sequence space and the forward shift map $\sigma^{+}$on it. They constitute a dynamical system $\left(\sum_{m}^{+}, \sigma^{+}\right)$which is a generalization of the frequently used system $\left(\Sigma_{2}, \sigma\right)$ where $\sum_{2}$ is the space of sequences consisting of the binary symbols 0 and 1 . The symbolic dynamics, so the name goes in literatures, has many applications with much of it in the application in coding [1]. Further, the shift map $\sigma$ has many properties for which they have been studied in a good number of papers in recent times $[2,3,4]$. The symbol space we use here has the structure of a metric which is defined in a natural way. So the study can be related to the metric space with the advantage that we can use the standard concepts of dynamical systems available therein. Our purpose is to study the chaotic and related properties of the shift map. There are several notions of chaoticity defined on metric spaces.

Biswas H. R. [5] discussed the concept of the generalized shift map $\sigma_{n}$ in the symbol space $\Sigma_{2}$ and proved some chaotic properties of the generalized shift map $\sigma_{\mathrm{n}}$ such as chaotic dependence on initial conditions, topologically transitive, totally transitive under a new definition of chaos. Ju H., Shao H., Choe Y. and Shi Y. [6] explained conditions for maps to be topologically conjugate or semi-conjugate to subshifts of finite type and criteria of chaos and we get basic idea about shift map and cantor set of logistic function from [7].

In Section 2, we describe the mathematical preliminaries which are requirements for the subsequent chapters. In Section 3 , we have proved that the forward shift map $\sigma^{+}$is topologically transitive and topologically mixing. We observed that the forward shift map $\sigma^{+}$on $\sum_{m}^{+}$is generically $\delta$-chaotic with $\delta=\operatorname{diam}\left(\sum_{m}^{+}\right)=1$. We also proved that the dynamical systems $\left(\sum_{m}^{+}, \sigma^{+}\right)$has chaotic dependence on initial conditions. 
In Section 4, we have established that the forward shift map on the generalized $m$-symbol space is DevC (Devaney chaotic) and as a consequence it is chaotic in Auslander-Yorke's sense. Further it is proved that is EDevC (Exact Devaney chaotic) and consequently MDevC (Mixing Devaney chaotic) and WMDevC (Weakly mixing Devaney chaotic) map. We have proved that $\sigma^{+}: \sum_{m}^{+} \rightarrow \sum_{m}^{+}$is topologically conjugate to the map $f_{m}(x)=\operatorname{mx}(\bmod 1)$ on the space $R / Z$. Finally, we have given a counter example that not all topologically transitive maps are totally transitive and given an example of a continuous function which is topologically transitive but not chaotic.

\section{Mathematical Preliminaries}

In 1975, Li and Yorke [8] gave the first mathematical definition of chaos through the introduction of $\delta$-scrambled sets. In 1980, J. Auslander and J. A. Yorke [9] defined chaos by associating the concept of transitivity and sensitivity which is known as Auslander-Yorke chaos. Thereafter, in 1989, R. L. Devaney [10] defined chaos in a very general way. It has three components described in the following three definitions.

Definition 2.1 (Li-Yorke Pair) [8]:

A pair $(x, y) \in X^{2}$ is called a Li-Yorke pair with modulus $\delta>0$ if $\lim _{n \rightarrow \infty} \sup d\left(f^{n}(x), f^{n}(y)\right) \geq \delta$ and $\lim _{n \rightarrow \infty} \inf d\left(f^{n}(x), f^{n}(y)\right)=0$, where $f$ is a continuous transformation on the compact metric space $(X, d)$. The set of all Li-Yorke pairs of modulus $\delta>0$ is denoted by $L Y(f, \delta)$.

Definition 2.2 (Auslander-Yorke Chaotic maps) [9]:

A continuous map $f$ on a metric space $(X, d)$ is said to be chaotic according to Auslander and York if $f$ is transitive and $f$ has sensitive dependence on initial conditions. So it follows that a Devaney Chaotic map (DevC) map is always chaotic in Auslander-Yorke's sense.

Definition 2.3 (Sensitive Dependence on initial conditions) [10]:

Let $(X, d)$ be a metric space. A continuous map $f: X \rightarrow X$ has sensitive dependence on initial conditions if there exists $\delta>0$ such that, for any $x \in X$ and any neighborhood $N(x)$ of $x$, there exists $y \in N(x)$ and $n \geq 0$ such that $d\left(f^{n}(x), f^{n}(y)\right)>\delta$.

Definition 2.4 (Topologically transitive) [10]:

Let $(X, d)$ be a metric space. A map $f: X \rightarrow X$ is topologically transitiveif for every pair of non-empty open sets $U$ and $V$ of $X$, there exists $n \in N$ such that $f^{n}(U) \cap V \neq \varnothing$.

Definition 2.5 (Periodic point) [10]:

Let $f: X \rightarrow X$ be a mapping where $X$ is any nonempty set. A point $z \in X$ is a periodic point if $f^{k} z=z$ for some positive integer $k$.

Definition 2.6 (Devaney’s chaos) [10]:

Let $(X, d)$ be a metric space. A map $f: X \rightarrow X$ is said to be chaotic in the sense of Devaney if the following three properties hold:

(i) $f$ has sensitive dependence on initial conditions;

(ii) $f$ is topologically transitive and

(iii) periodic points are dense in $X$.

Subsequently, in Banks et al. [11], it was shown that topological transitivity and dense periodic orbits together imply sensitive dependence on initial conditions. So the condition (i) of Devaney's definition is redundant. It is also known that in an interval (not necessarily finite) a continuous, topological transitive map is chaotic in the sense of Devaney [10].

Devaney's chaos is important from analytical as well as an application point of view. In the year 1992, L. Snoha [12] introduced the concept of dense chaos. Today, Devaney chaos has been studied in more extensive ways that is with much stronger conditions leading to exact Devaney chaos, mixing Devaney chaos, weak mixing Devaney chaos, etc. A Devaney chaotic map $f: X \rightarrow X$ is said to exhibit exact Devaney chaos (EDevC) property [10] if it is exact, that is, if for every non-empty open set $U$ of $X$, there exists some $m \in N$ such that $f^{m}(U)=X$. On the other hand, a Devaney chaotic map $f: X \rightarrow X$ is said to exhibit mixing Devaney chaos (MDevC), if it is mixing, that is, for any pair of non-empty open sets $U$ and $V$ of $X, \exists n_{0} \in N$ such that $f^{n}(U) \cap V \neq \emptyset \forall n \geq n_{0}$. Further, the Devaney chaotic map $f: X \rightarrow X$ is said to exhibit weak mixing Devaney chaos (WMDevC), if it is weak mixing that is, $f \times f$ is transitive on $X \times X$ that is for every non-empty open set of $U \times V$ of $X \times X, \exists n \in N$ such that $f^{n}(U) \cap V \neq \emptyset$. Sensitive dependence on initial conditions is an important property for any chaotic map. Bau - Sen Du [4] gave a new strong definition of chaos by using shift map in the symbol space $\Sigma_{2}$, and by considering a dense uncountable invariant scrambled set in $\Sigma_{2}$.

In this paper, we work on a one-sided $m$-symbol sequence space which is

$\sum_{m}^{+}=\{0,1,2, \ldots \ldots m-1\}^{N}=\left\{\left(x_{i}\right)_{i=1}^{\infty}: x_{i} \in\{0,1,2 \ldots \ldots m-1\}, m \in N\right\}$, and discuss the Devaney chaoticity of the forward shift map $\sigma^{+}: \sum_{m}^{+} \rightarrow \sum_{m}^{+}$. Many works have been done on this system for the particular case of $m=2$. Several aspects of the binary sequence space $\sum_{2}^{+}$and the forward shift map $\sigma^{+}$on it can be found in various papers and books such 
as $[1,2,4,5,14,22,23,24]$. Since $\sum_{2}^{+}$is a special case of $\sum_{m}^{+}$, our results are also valid in $\left(\sum_{2}^{+}, \sigma^{+}\right)$. Further it may be mentioned that our study falls in the greater domain of chaotic dynamics. Some references from this expanding area of research are noted in [17, 19, 21, 22].

Definition 2.7 (Topological Conjugacy) [10]:

Let $f: X \rightarrow X$ and $g: Y \rightarrow Y$ be two continuous maps on the metric spaces $X$ and $Y$. If there exists a homeomorphism $h: X \rightarrow Y$ such that $h o f=g o h$, then $f$ is said to be topologically conjugate to the map $g$. In this case, $h$ is called a topological conjugacy [10]. On the other hand, if for the continuous maps $f: X \rightarrow X$ and $g: Y \rightarrow Y$, there exists a surjection $h: X \rightarrow Y$ such that $h o f=g o h$, then $f$ is topologically semi-conjugate to $g$.

The word conjugacy as referred to dynamical systems means the similarity between the dynamical behavior of two mappings. Topological conjugacy between maps is a very powerful tool in the study of dynamical systems. Topological conjugacy is an as important concept in the theory of dynamical systems as it is an important tool that can be used to make predictions about the behavior of a dynamical system by comparing it with another dynamical system whose specific properties are known.

Definition 2.8 (Generically $\boldsymbol{\delta}$-chaotic maps) [14]:

The continuous map $f: X \rightarrow X$ on a compact metric space $X$ is called generically $\delta$-chaotic if $L Y(f, \delta)$ is residual in $X^{2}$.

Definition 2.9 (Topologically mixing) [15]:

Let $f: X \rightarrow X$ be a map on a topological space $(X, T)$ and $f$ is topologically mixing if given nonempty open sets $U, V \subset X$, there exists $n_{0}$ such that $f^{n}(U) \cap V \neq \varnothing$ for all $n \geq n_{0}$.

Definition 2.10 (Weakly topologically mixing) [15]:

Let $f: X \rightarrow X$ be a map on a topological space $(X, T)$ and $f$ is weakly topologically mixing if for every nonempty pair $U, V \subset X$, there exists a positive integer $k$ such that $f^{k}(U) \cap V \neq \varnothing$.

Note: From the above two definitions it follows that topologically mixing property implies weakly topologically mixing property.

Definition 2.11 (Chaotic dependence on initial conditions) [16]:

A dynamical system $(X, f)$ is said to have chaotic dependence on initial conditions if for any $x \in X$ and every neighborhood $N(x)$ of $x$ there is a $y \in N(x)$ such that the pair $(x, y) \in X^{2}$ is Li-Yorke.

Definition 2.12 (Weak and modified weak chaotic dependence on initial conditions) [16]: A dynamical systems $(X, f)$ is said to have weak chaotic dependence on initial conditions if for any $x \in X$ and every neighbourhood $N(x)$ of $x$ there are $y, z \in N(X)$ [modified weak case $y \neq x, z \neq x$ ] such that $(y, z) \in X^{2}$ is Li-Yorke.

We also need the following Proposition, Lemma, and Theorem.

Proposition 2.1: Let $T: X \rightarrow X$ be a continuous map on a compact metric space $X$. If $T$ is topologically weak mixing, then it is generically $\delta$-chaotic on $X$ with $\delta=\operatorname{diam}(X)$.

Lemma 2.1: Let $s, t \in \Sigma_{2}$ and $s_{i}=t_{i}, i=0,1, \ldots \ldots \ldots, m$. Then, $d(s, t)<\frac{1}{2^{m}}$ and conversely if $d(s, t)<\frac{1}{2^{m}}$ then, $s_{i}=t_{i}$, for $i=0,1, \ldots \ldots \ldots, m$.

Theorem 2.1: The forward shift map $\left(\sum_{m}^{+}, \sigma^{+}\right)$is topological dynamical systems (TDS).

Proof: It is immediate that the space $\sum_{m}^{+}=\{0,1,2, \ldots \ldots, m-1\}^{N}=\left\{x=\left(x_{i}\right)_{i=1}^{\infty}: x_{i} \in\{0,1,2, \ldots \ldots, m-1\}\right\}$ where $m(\geq 2) \in N$, the generalized $m$-symbol sequence space, is a compact metric space under the distance function $d: \sum_{m}^{+} \times \sum_{m}^{+} \rightarrow R$ defined by $d(x, y)=\sum_{k \geq 1} \frac{\left|x_{k}-y_{k}\right|}{m^{k}}$ for $x=\left(x_{1}, x_{2}, x_{3}, \ldots \ldots\right), y=\left(y_{1}, y_{2}, y_{3}, \ldots \ldots\right) \in \sum_{m}^{+}$. Again, it is obvious that the forward shift transformation $\sigma^{+}: \sum_{m}^{+} \rightarrow \sum_{m}^{+}$defined by $\sigma\left(x_{1}, x_{2}, x_{3}, \ldots \ldots\right)=\left(x_{2}, x_{3}, x_{4}, \ldots \ldots\right)$ is continuous.

Therefore, $\left(\sum_{m}^{+}, \sigma^{+}\right)$is clearly a topological dynamical systems.

Note: One important observation is that for any $m, n(n<m) \in N$, we always have that $\sum_{n}^{+} \subseteq \sum_{m}^{+}$. So whatever is true for $\sum_{m}^{+}$is also true for $\sum_{n}^{+}$.

The following example of a dynamical system which will be useful for us.

Example 2.1: Consider the map $f_{m}(x)=m x(\bmod 1)$ and the unit circle $S^{1}$. We have to show that $\left(S^{1}, f_{m}\right)$ is a topological dynamical systems.

Proof: It is a well-known fact that for any $m(\geq 2) \in N$, the map $f_{m}:[0,1] \rightarrow[0,1]$ such that. $f_{m}(x)=\operatorname{mx}(\bmod 1)$ is discontinuous at the points $\frac{1}{m}, \frac{2}{m}, \frac{3}{m}, \ldots, \frac{m-1}{m} \in I$. This map is well defined on $R / Z \cong I / \sim$ where $R / Z$ is the space of equivalence classes $x+Z$ of real numbers $x$ up to integers such that two real numbers $x, y$ belong to the same equivalence class if and only if $\exists$ an integer $k \in Z$ such that $x=y+k$ and $I / \sim$ denotes the unit interval with the endpoints identified such that the symbol $\sim$ implies that $0 \sim 1$ are glued together to get a circle. $R / Z$ is a metric space under the metric $d(x, y)=\min _{m \in z}|x-y+m|$. 
Also, the unit circle $S^{1}=\{z \in C:|z|=1\}=\left\{e^{2 \pi i \theta}: 0 \leq \theta \leq 1\right\}$ is a metric space with respect to the modified arc length distance $d$ defined by

$$
d\left(e^{2 \pi i \theta_{1}}, e^{2 \pi i \theta_{2}}\right)=\left|\theta_{2}-\theta_{1}\right| \text { or }\left[1-\left|\theta_{2}-\theta_{1}\right|\right] \text { according to }\left|\theta_{2}-\theta_{1}\right| \leq \frac{1}{2} \text { or }\left|\theta_{2}-\theta_{1}\right|>\frac{1}{2} \text {. }
$$

$S^{1}$ is a compact metric space and there is a one-to-one correspondence between $R / Z$ and $S^{1}$ via the map $\Psi: R / Z \rightarrow S^{1}$ given by $\Psi(x)=e^{2 \pi i x}$.

Thus $R / Z$ is identified with $S^{1}$ via the 1-1 correspondence $\Psi(x)=e^{2 \pi i x}$. So the map $f_{m}(x)$ on $R / Z \cong I / \sim$ can be defined on $S^{1}$ as $f_{m}\left(z=e^{2 \pi i x}\right)=e^{2 \pi i(m x)}=\left(e^{2 \pi i x}\right)^{m}=z^{m}$. This is a contradiction map on $S^{1}$. Ultimately, $\left(S^{1}, f_{m}\right)$ is a topological dynamical systems.

\section{Chaotic properties of $\sigma^{+}$:}

In this section, we prove some chaotic properties of the forward shift map $\sigma^{+}$.

Theorem 3.1: [The Proximity Theorem]

Let $x, y \in \sum_{m}^{+}$. Then $d(x, y) \leq \frac{1}{m^{n}}$ if and only if $x$ and $y$ agree up to $n$-digits.

Proof: Let $x=\left(x_{1}, x_{2}, x_{3}, \ldots \ldots\right), y=\left(y_{1}, y_{2}, y_{3}, \ldots \ldots\right) \in \sum_{m}^{+}$and $x$ and $y$ agree up to the $n$-digits.

Then $x_{i}-y_{i}=0$ for $i=1,2,3, \ldots \ldots, n$ and therefore we have $\sum_{i=1}^{n} \frac{\left|x_{i}-y_{i}\right|}{m^{i}}=0$.

So $d(x, y)=\sum_{k \geq 1} \frac{\left|x_{k}-y_{k}\right|}{m^{k}}=\sum_{i=1}^{n} \frac{\left|x_{i}-y_{i}\right|}{m^{i}}+\sum_{k>n} \frac{\left|x_{k}-y_{k}\right|}{m^{k}}=0+\sum_{k>n} \frac{\left|x_{k}-y_{k}\right|}{m^{k}}=\sum_{k>n} \frac{\left|x_{k}-y_{k}\right|}{m^{k}}$.

Now, $x=\left(x_{1}, x_{2}, x_{3}, \ldots \ldots\right), y=\left(y_{1}, y_{2}, y_{3}, \ldots \ldots\right) \in \sum_{m}^{+}$

$\Rightarrow x_{i}, y_{i} \in\{0,1,2,3, \ldots, m-1\}, \forall i \in N$

$\Rightarrow\left|x_{i}-y_{i}\right| \leq m-1, \quad \forall i \in N$

$\Rightarrow \frac{\left|x_{k}-y_{k}\right|}{m^{k}} \leq \frac{m-1}{m^{k}}, \forall k>n$

$\therefore d(x, y)=\sum_{k>n} \frac{\left|x_{k}-y_{k}\right|}{m^{k}} \leq \sum_{k=n+1}^{\infty} \frac{m-1}{m^{k}}=\frac{m-1}{m^{n+1}} \sum_{r=0}^{\infty} \frac{1}{m^{r}}=\frac{m-1}{m^{n+1}} \cdot \frac{1}{1-\frac{1}{m}}=\frac{1}{m^{n}}$.

Conversely, let $(x, y) \leq \frac{1}{m^{n}}$. We need to show that $x$ and $y$ agree up to $n$-digits.

If possible, let $x$ disagrees to $y$ at least at one digit that precedes the $n^{\text {th }}$ digit, say at $i^{\text {th }}$ digit where $1 \leq i \leq n-1$ and agrees at all other digits up to $n$-digits. Then

[ Since $i \leq n-1<n]$.

$$
d(x, y)=\sum_{k \geq 1} \frac{\left|x_{k}-y_{k}\right|}{m^{k}}=\frac{\left|x_{i}-y_{i}\right|}{m^{i}}+\sum_{k=n+1}^{\infty} \frac{\left|x_{k}-y_{k}\right|}{m^{k}} \geq \frac{\left|x_{i}-y_{i}\right|}{m^{i}} \geq \frac{1}{m^{i}}>\frac{1}{m^{n}}
$$

This contradicts our assumption that $d(x, y)<\frac{1}{m^{n}}$. Further, if $x$ disagrees to $y$ at more than one digit for $1 \leq i \leq n-1$, then also proceeding as above we get contradictions. So it follows that $x$ and $y$ must agree up to $n$-digit.

Theorem 3.2: The forward shift map $\sigma^{+}: \sum_{m}^{+} \rightarrow \sum_{m}^{+}$is topologically transitive.

Proof: To establish that the forward shift map $\sigma^{+}$is topologically transitive, we need to show that for any two non-empty open sets $U$ and $V$ of $\sum_{m}^{+}$, there exists $n \in N$ such that $\sigma^{n}(U) \cap V \neq \varnothing$.

Let $x=\left(x_{1}, x_{2}, x_{3}, \ldots \ldots\right) \in U$ and $y=\left(y_{1}, y_{2}, y_{3}, \ldots \ldots\right) \in V$ be arbitrary (since $U$ and $V$ are non-empty open sets, so we always have such points).

Now, $x \in U, y \in V$ and $U, V$ are open sets. So, $\exists$ open balls $B\left(x, r_{1}\right) \subseteq U$ and $B\left(y, r_{2}\right) \subseteq V$. If $r=\min \left\{r_{1}, r_{2}\right\}$, then $B(x, r) \subseteq U$ and $B(y, r) \subseteq V$. We choose $n \in N$ such that $\frac{1}{m^{n}}<r$. Consider the point,

$z=\left(x_{1}, x_{2}, x_{3}, \ldots \ldots x_{n}, y_{1}, y_{2}, y_{3}, \ldots \ldots\right) \in \sum_{m}^{+}$which agrees with $x$ up to the $n^{\text {th }}$ term. Therefore, by Proximity theorem, we have that,

$d(x, z) \leq \frac{1}{m^{n}}<r \Rightarrow z \in B(x, r) \subseteq U$ and consequently it follows that $\sigma^{n}(z) \in \sigma^{n}(U)$.

Also, $\sigma^{n}(z)=\left(y_{1}, y_{2}, y_{3}, \ldots \ldots\right)=y \in V, \quad y=\sigma^{n}(z) \in \sigma^{n}(U) \Rightarrow y=\sigma^{n}(z) \in \sigma^{n}(U) \cap V$. So it follows that $\sigma^{n}(U) \cap V \neq \varnothing$ and hence $\sigma^{+}: \sum_{m}^{+} \rightarrow \sum_{m}^{+}$is topologically transitive.

Theorem 3.3: The forward shift map $\sigma^{+}: \sum_{m}^{+} \rightarrow \sum_{m}^{+}$is topologically mixing.

Proof: Let $U$ and $V$ be any two non-empty open sets in $\sum_{m}^{+}$. We show that there exists a non-negative integer $n_{0}$ such that $\sigma^{n}(U) \cap V \neq \varnothing, \forall n \geq n_{0}$.

Let $x=\left(x_{1}, x_{2}, x_{3}, \ldots \ldots\right) \in U$ and $y=\left(y_{1}, y_{2}, y_{3}, \ldots \ldots\right) \in V$ be arbitrary (since $U$ and $V$ are non-empty, so we must have such points in $U$ and $V$ ). Then, since $x \in U, y \in V$ and $U, V$ are open sets in $\sum_{m}^{+}$, $\exists$ open balls $B\left(x, r_{1}\right), B\left(y, r_{2}\right)$ such that $B\left(x, r_{1}\right) \subseteq U$ and $B\left(y, r_{2}\right) \subseteq V$. 
If $r=\min \left\{r_{1}, r_{2}\right\}$, then $B(x, r) \subseteq U$ and $B(y, r) \subseteq V$ and choose $k \in N$ such that $\frac{1}{m^{k}}<r$. We then construct a sequence $\left\{z_{n}\right\}$ of points in $\sum_{m}^{+}$with the help of $k, x$ and $y$ such that,

$$
\begin{aligned}
& z_{1}=\left(x_{1}, x_{2}, x_{3}, x_{4} \ldots x_{k}, y_{1}, y_{2}, y_{3}, y_{4} \ldots\right), \\
& z_{2}=\left(x_{1}, x_{2}, x_{3}, x_{4} \ldots x_{k}, a_{1}, y_{1}, y_{2}, y_{3}, y_{4} \ldots\right), \\
& z_{3}=\left(x_{1}, x_{2}, x_{3}, x_{4} \ldots x_{k}, a_{1}, a_{2}, y_{1}, y_{2}, y_{3}, y_{4} \ldots\right), \ldots, \\
& z_{i}=\left(x_{1}, x_{2}, x_{3}, x_{4} \ldots x_{k}, a_{1}, a_{2}, \ldots \ldots, a_{i-1}, y_{1}, y_{2}, y_{3}, y_{4} \ldots \ldots\right), i \geq 2,
\end{aligned}
$$

$a_{i} \in\{0,1,2, \ldots \ldots, m-1\}$.

Here, every $z_{i}, i \geq 2$ is constructed by using the finite word obtained by taking first (i-1) consecutive symbols of a fixed sequence $a=\left(a_{1}, a_{2}, a_{3}, \ldots \ldots a_{i-1}, \ldots\right) \in \sum_{m}^{+}$chosen arbitrarily. More precisely, the first $k$ letters of $z_{i}$, for each $i \geq 2$, is the finite word,

$x_{[1, k]}=\left(x_{1}, x_{2}, x_{3}, x_{4} \ldots \ldots x_{k}\right)$ taken from $x \in U$ and then follows the word.

$a_{[1, i-1]}=\left(a_{1}, a_{2}, a_{3}, \ldots \ldots, a_{i-1}\right)$ taken from $a$ and at last the sequence representing $y$, i.e., $z_{i}=\left(x_{[1, k]}, a_{[1, i-1]}, y\right)$. In this case, we can also use $a$ fixed letter from the alphabet set $\{0,1,2, \ldots, m-1\}$ repeating it for $(i-1)$ times rather than using $a_{[1, i-1]}$.

Now, by using the Proximity theorem, we have, $d\left(x, z_{i}\right) \leq \frac{1}{m^{k}}<r$ [since $x$ and $z_{i}$ agree up to the $k$ th digits], for all $i \in N$. So, $z_{i} \in B(x, r) \subseteq U$ and hence, $\sigma^{k+i-1}\left(z_{i}\right) \in \sigma^{k+i-1}(B(x, r)) \subseteq \sigma^{k+i-1}(U)$ for all $i \in N$.

Also, $\sigma^{k+i-1}\left(z_{i}\right)=\left(y_{1}, y_{2}, y_{3, \ldots \ldots . . .}\right) \in V, \sigma^{k+i-1}\left(z_{i}\right) \in \sigma^{k+i-1}(U)$ imply that $\sigma^{k+i-1}(U) \cap V \neq \emptyset$, for all $i \geq 2$.

Therefore, $\sigma^{n}(U) \cap V \neq \varnothing$, for all $n \geq k$.

Hence, the forward shift map $\sigma^{+}: \sum_{m}^{+} \rightarrow \sum_{m}^{+}$is topologically mixing.

Theorem 3.4: The forward shift map $\sigma^{+}: \sum_{m}^{+} \rightarrow \sum_{m}^{+}$has sensitive dependence on initial conditions.

Proof: Let $x \in \sum_{m}^{+}$be arbitrary and $N(x)$ be an arbitrary neighbourhood of $x$. Then by definition of a neighbourhood, there exists a non-empty open set $H$ such that $x \in H \subseteq N(x)$. Now $x \in H, H$ is open in $\sum_{m}^{+}, \exists$ an open ball $B(x, r)$ such that $B(x, r) \subseteq H \subseteq N(x)$. Let $y \in B(x, r) \subseteq H \subseteq N(x)$ such that $x \neq y$ and $x$ is very close to $y$. This is always possible to have a very close point to $x$, because we can choose a $k \in N$ as large as we want to satisfy $\frac{1}{m^{k}}<r$ and for this large $k \in N$ we can construct the point $y$ in such a way that this agrees with $x$ up to $k$-digits. Then, $d(x, y) \leq \frac{1}{m^{k}}<r$ and hence for large value of $k, x$ will be too close to $y$.

Let $d(x, y)=\varepsilon$. Then, since $x$ is very close to $y$ and $\varepsilon$ is very small, depending on the value of $\varepsilon>0, \exists$ a large and unique $n \in N$ such that $\frac{1}{m^{n+1}}<\varepsilon \leq \frac{1}{m^{n}}$, Consider $d(x, y)=\varepsilon \leq \frac{1}{m^{n}}$.

Then, $d(x, y) \leq \frac{1}{m^{n}} \Rightarrow x$ and $y$ agree up to the $n^{\text {th }}$ digit

$\Rightarrow(n+1)^{\text {th }}$ digits of $x$ and $y$ are different

$\Rightarrow$ The first digit of $\sigma^{n}(x)$ and $\sigma^{n}(y)$ are different

$\Rightarrow d\left(\sigma^{n}(x), \sigma^{n}(y)\right)=\sum_{i=1}^{\infty} \frac{\left|x_{n+i}-y_{n+i}\right|}{m^{i}}=\frac{\left|x_{n+1}-y_{n+1}\right|}{m}+\sum_{i=2}^{\infty} \frac{\left|x_{n+i}-y_{n+i}\right|}{m^{i}} \geq \frac{1}{m}$ From the above relation, it is clear that $\frac{1}{m}$ plays the role of sensitivity constant $\delta$.

Thus, for every $x \in \sum_{m}^{+}$and any neighbourhood $N(x)$ of $x, \exists y \in N(x)$ and $n>0$ satisfying $d\left(\sigma^{n}(x), \sigma^{n}(y)\right) \geq$ $\delta$ for $\delta=\frac{1}{m}$.

Hence the forward shift transformation $\sigma^{+}: \sum_{m}^{+} \rightarrow \sum_{m}^{+}$has sensitive dependence on initial conditions.

Theorem 3.5: The set $P\left(\sigma^{+}\right)$, the set of all the periodic points of the forward shift map $\sigma^{+}$, is dense in $\sum_{m}^{+}$.

Proof: We first show that $\sigma^{+}$has $m^{n}-m$ periodic points of period- $n$ in $\sum_{m}^{+}$for $n \geq 2$. It is to be noted that if a definite block of $n$-digits from the set $\{0,1,2,3,4, \ldots \ldots, m-1\}$ repeats indefinitely, then it is a periodic point of $\sigma$ of period- $n$ in $\sum_{m}^{+}$. A block of $n$-digits can be formed with the $m$ distinct digits $0,1,2,3, \ldots, m-1$ in $m^{n}$ ways. These blocks contain the $m$-blocks formed by the same digit which are not periodic points of period- $n$. In fact, periodic points of period-1 i.e., fixed points. So, we have only $\left(m^{n}-m\right)$ numbers of periodic points of period- $n$ in $\sum_{m}^{+}$.

Consider an arbitrary point $x \in \sum_{m}^{+}$. We show that for any $\varepsilon>0$, however small, there is a point $p \in P\left(\sigma^{+}\right)$such that $d(x, p)<\varepsilon$. Let $x=\left(x_{1}, x_{2}, x_{3}, \ldots \ldots\right)$, for the fixed small $\varepsilon>0$, we can always find a positive integer $n \in N$ such that $\frac{1}{m^{n}}<\varepsilon$.

Now, we construct a periodic point $p \in P\left(\sigma^{+}\right)$of periodic $(n+1)$ such that, $p=\left(x_{1}, x_{2}, x_{3}, \ldots \ldots x_{n}, y, x_{1}, x_{2}, x_{3}, \ldots \ldots . x_{n}, y, x_{1}, x_{2}, x_{3}, \ldots \ldots x_{n}, y, \ldots\right)$ i.e., $p$ is constructed by repeating the word $W=\left(x_{1}, x_{2}, x_{3}, \ldots \ldots x_{n}, y\right)$ infinite number of times so that it agrees with the digits of $x$ up to $n$-terms and disagrees at 
$(n+1)$ th digit such that $x_{n+1} \neq y$ and, $d(x, p) \leq \frac{1}{m^{n}}<\varepsilon$.

Thus, for every $x \in \sum_{m}^{+}$and $\varepsilon>0, \exists p \in P\left(\sigma^{+}\right)$such that $d(x, p)<\varepsilon$. That is, however small $\varepsilon>0$ may be for any $x \in \sum_{m}^{+}$there is always a point $p \in P\left(\sigma^{+}\right)$which is at a distance less than the arbitrarily small quantity $\varepsilon>0$. Hence, the set $P\left(\sigma^{+}\right)$is dense.

Theorem 3.6: The forward shift map $\sigma^{+}$on $\sum_{m}^{+}$is Devaney as well as Auslander-Yorke chaotic.

Proof: We have already proved that $\sigma^{+}$is topologically transitive, it has sensitive dependence on initial conditions and the set of all the periodic points of $\sigma^{+}$is dense in $\sum_{m}^{+}$. That is, $\sigma^{+}$satisfies all the requirements for Devaney as well as Auslander-Yorke chaoticity. So it is Devaney as well as Auslander-Yorke chaotic [9].

Theorem 3.7: The forward shift map $\sigma^{+}$on $\sum_{m}^{+}$is generically $\delta$-chaotic with $\delta=\operatorname{diam}\left(\sum_{m}^{+}\right)=1$.

Proof: We have established that the forward shift transformation $\sigma^{+}$on $\sum_{m}^{+}$is topologically mixing. Since by Definition 2.5 and 2.6, a continuous topologically mixing map on a compact metric space is also topologically mixing map on a compact metric space and is also topologically weak mixing, so the forward shift transformation $\sigma^{+}$being a continuous topologically mixing map on the compact metric space $\Sigma_{m}^{+}$is topologically weak mixing. Also, using Proposition 2.1, we see that a continuous topologically weak mixing map on a compact metric space $X$ is generically $\delta$-chaotic on $X$ with $\delta=\operatorname{diam}(X)$. So it follows that the forward shift transformation $\sigma^{+}$on $\sum_{m}^{+}$is generically $\delta$-chaotic with

$\delta=\operatorname{diam}\left(\sum_{m}^{+}\right)=1$.

Theorem 3.8: The dynamical system $\left(\sum_{m}^{+}, \sigma^{+}\right)$has chaotic dependence on initial conditions.

Proof: Let $a=\left(a_{1}, a_{2}, a_{3}, \ldots \ldots\right) \in \sum_{m}^{+}$be an arbitrary point and $N(a)$ be any neighbourhood of $a$. Then, there exists an open set (open neighbourhood) $U$ of $\sum_{m}^{+}$such that $a \in U \subseteq N(a)$.

Now since $a \in U$ and $U$ is an open set, so there exists an open ball $B(a, r)$ with some radius $r>0$ s.t. $B(a, r) \subseteq U \subseteq$ $N(a)$. Then for this $r>0$ we can choose a sufficiently large positive integer $\mathrm{n}$ such that $\frac{1}{\mathrm{~m}^{n}}<r$. We now find a point $b \in B(a, r) \subseteq U \subseteq N(a)$ such that the pair $(a, b) \in \sum_{m}^{2}$ is Li-Yorke.

Using the letters in $a=\left(a_{1}, a_{2}, a_{3}, \ldots \ldots\right) \in \sum_{m}^{+}$, we define the words $W(a, 3 n), W(a, 5 n), W(a, 7 n), \ldots \ldots$ etc. as follows:

and so on.

$$
\begin{aligned}
& W(a, 3 n)=\left(a_{3 n+1}^{*}, a_{3 n+2}^{*}, \ldots, a_{4 n}^{*}, a_{4 n+1}, a_{4 n+2}, \ldots, a_{5 n}\right), \\
& W(a, 5 n)=\left(a_{5 n+1}^{*}, a_{5 n+2}^{*}, \ldots, a_{6 n}^{*}, a_{6 n+1}, a_{6 n+2}, \ldots, a_{7 n}\right), \\
& W(a, 7 n)=\left(a_{7 n+1}^{*}, a_{7 n+2}^{*}, \ldots, a_{8 n}^{*}, a_{8 n+1}, a_{8 n+2}, \ldots, a_{9 n}\right), \ldots
\end{aligned}
$$

Now using the above defined words we construct the point $b$ as follows:

$$
b=\left(a_{1}, a_{2}, a_{3}, \ldots, a_{n},\left(0^{*}\right)^{n},(0)^{n}, W(a, 3 n), W(a, 5 n), W(a, 7 n), W(a, 9 n), \ldots\right)
$$

where $\left(0^{*}\right)^{n}=0^{*}, 0^{*}, 0^{*}, \ldots 0^{*},(0)^{n}=0,0,0, \ldots, 0$ and $0^{*}=(m-1)-0=m-1$.

From the construction of $b$, it is clear that $b$ agrees with $a$ up to the $n^{\text {th }}$ term. So by Proximity theorem, we have $d(a, b) \leq \frac{1}{m^{n}}<r$ and hence, $b \in B(a, r) \subseteq U \subseteq N(a)$. Here, we see that $b$ contains infinitely many words of the type $W(a,(2 k-1) n)$ containing $2 n$ letters each where $k \geq 2$ is an integer.

Also,

$$
\begin{aligned}
& \sigma^{3 n}(b)=\left(a_{3 n+1}^{*}, a_{3 n+2}^{*}, \ldots, a_{4 n}^{*}, a_{4 n+1}, a_{4 n+2}, \ldots, a_{5 n}, a_{5 n+1}^{*}, a_{5 n+2}^{*}, \ldots\right) \\
& \sigma^{4 n}(b)=\left(a_{4 n+1}, a_{4 n+2}, \ldots, a_{5 n}, a_{5 n+1}^{*}, a_{5 n+2}^{*}, \ldots, a_{6 n}^{*}, a_{6 n+1}, a_{6 n+2}, \ldots\right)
\end{aligned}
$$

Therefore, $\operatorname{supd}\left(\sigma^{n}(a), \sigma^{n}(b)\right) \geq d\left(\sigma^{3 n}(a), \sigma^{3 n}(b)\right)$ and so

$\lim _{n \rightarrow \infty} \operatorname{supd}\left(\sigma^{n}(a), \sigma^{n}(b)\right) \geq \lim _{n \rightarrow \infty} d\left(\sigma^{3 n}(a), \sigma^{3 n}(b)\right) \geq \lim _{n \rightarrow \infty} \sum_{r=1}^{n} \frac{\left|a_{3 n+r}-a_{3 n+r}^{*}\right|}{m^{r}}$

$\geq \lim _{n \rightarrow \infty}\left\{\frac{1}{m}+\frac{1}{m^{2}}+\cdots+\frac{1}{m^{n}}\right\}=\frac{1}{m-1}$

Again, $0 \leq \lim _{n \rightarrow \infty}$ inf $d\left(\sigma^{n}(a), \sigma^{n}(b)\right) \leq \lim _{n \rightarrow \infty} d\left(\sigma^{4 n}(a), \sigma^{4 n}(b)\right)$

$=\lim _{n \rightarrow \infty} d\left(\left(a_{4 n+1}, \ldots, a_{5 n}, a_{5 n+1}, \ldots, a_{6 n}, a_{6 n+1}, \ldots\right),\left(a_{4 n+1}, \ldots, a_{5 n}, a_{5 n+1}^{*}, a_{5 n+2}^{*}, \ldots \ldots, a_{6 n}^{*}, a_{6 n+1}, \ldots \ldots\right)\right)$

$\leq \lim _{n \rightarrow \infty}\left\{\left(\frac{m-1}{m^{n+1}}+\frac{m-1}{m^{n+2}}+\cdots+\frac{m-1}{m^{2 n}}\right)+\left(\frac{m-1}{m^{3 n+1}}+\frac{m-1}{m^{3 n+2}}+\cdots+\frac{m-1}{m^{4 n}}\right)+\cdots\right\}$

$=\lim _{n \rightarrow \infty}\left\{\left(\frac{m-1}{m}+\frac{m-1}{m^{2}}+\cdots+\frac{m-1}{m^{n}}\right) \cdot\left(\frac{1}{m^{n}}+\frac{1}{m^{3 n}}+\frac{1}{m^{5 n}}+\cdots\right)\right\}$

$=\lim _{n \rightarrow \infty}\left\{\left(1-\frac{1}{m^{n}}\right) \cdot \frac{1}{m^{n}}\left(1+\frac{1}{m^{2 n}}+\frac{1}{m^{4 n}}+\frac{1}{m^{6 n}} \ldots \ldots\right)\right\}$ 


$$
\begin{aligned}
& =\lim _{n \rightarrow \infty}\left\{\left(1-\frac{1}{m^{n}}\right) \cdot \frac{1}{m^{n}} \cdot \frac{1}{1-\frac{1}{m^{2 n}}}\right\} \\
& =(1-0) \cdot 0 \cdot\left(\frac{1}{1-0}\right)=0
\end{aligned}
$$

Now, $0 \leq \lim _{n \rightarrow \infty} \operatorname{infd}\left(\sigma^{n}(a), \sigma^{n}(b)\right) \leq 0 \Rightarrow \lim _{n \rightarrow \infty} \operatorname{infd}\left(\sigma^{n}(a), \sigma^{n}(b)\right)=0$.

So, it follows that $\lim _{n \rightarrow \infty} \operatorname{supd}\left(\sigma^{n}(a), \sigma^{n}(b)\right) \geq \frac{1}{m-1}$ and $\lim _{n \rightarrow \infty} \operatorname{infd}\left(\sigma^{n}(a), \sigma^{n}(b)\right)=0$.

Hence, $(a, b) \in \sum_{m}^{2}$ is a Li-Yorke pair with modulus $\delta=\frac{1}{m-1}>0$. Therefore, the dynamical systems $\left(\sum_{m}^{+}, \sigma^{+}\right)$has chaotic dependence on initial conditions.

\section{Topological Conjugacy and Some Chaotic properties}

In this section, we use the concept of topological conjugacy to establish that the forward shift map on the generalized $m$-symbol space is Devaney chaotic and as a consequence, it is chaotic in Auslander-Yorke's sense. Further, it is proved that is Exact Devaney chaotic and consequently Mixing Devaney chaotic and Weakly mixing Devaney chaotic.

In the last part of this section, we give an example of a continuous function that is topologically transitive but not chaotic. We also give a suitable example illustrating that all topologically transitive maps are not totally transitive.

Theorem 4.1: If $f$ and $g$ are topologically conjugate via mapping $h$ and if $p$ is a fixed point of $f$, then $h(p)$ is a fixed point of $g$.

Proof: Since $h(p)=h(f(p))=\left(h^{\circ} f\right)(p)=\left(g^{\circ} h\right)(p)=g(h(p))$, it follows that $h(p)$ is a fixed point of $g$.

Theorem 4.2: If $f$ and $g$ are conjugate, the periodic points are carried into periodic points of the same periodic under conjugacy.

Proposition 4.1: If the TDS $f: X \rightarrow X$ is topologically conjugate to the TDS $g: Y \rightarrow Y$ by the conjugacy map $h: X \rightarrow Y$, then

(i) $f$ is topologically transitive if and only if $g$ is topologically transitive,

(ii) $f$ is DevC if and only if $g$ is DevC.

Theorem 4.3: Let $f$ and $g$ be topologically conjugate. Then, $f$ is chaotic if and only if $g$ is chaotic.

Proof: From the definition of chaos and using the Theorems 4.1 and 4.2, and Proposition 4.1, we observe that all the conditions in the definition of chaos are satisfied. Hence, it follows that if two mappings are topologically conjugate, then they have exactly the same dynamics, that is, their behavior regarding the number and nature of the fixed and periodic points is the same. Thus, if $f$ and $g$ be topologically conjugate, then $f$ is chaotic if and only if $g$ is chaotic.

Proposition 4.2: If the TDS $f: X \rightarrow X$ is topologically semi-conjugated to the TDS $g: Y \rightarrow Y$ then $f$ is topologically transitive implies $g$ is topologically transitive.

Theorem 4.4: The forward shift map $\sigma^{+}: \sum_{m}^{+} \rightarrow \sum_{m}^{+}$and the map $f_{m}: R / Z \rightarrow R / Z$ such that $f_{m}(x)=\operatorname{mx}(\bmod 1)$ are topologically semi-conjugated.

Note: The pre-image of every $m$-adic rational number in $R / Z=I / \sim$ is a set of two distinct sequences in $\sum_{m}^{+}$, one with a tail of 0 's and the other with a tail of (m-1)'s, so $\Psi$ is not injective. Hence, by restricting the domain of $\Psi$, we make $\Psi$ injective and thereby make it a conjugacy between $\sigma^{+}$and $f_{m}$.

Theorem 4.5: The forward shift map $\sigma^{+}: \sum_{m}^{+} / \sim \rightarrow \sum_{m}^{+} / \sim$ and the map $f_{m}(x)=\operatorname{mx}(\bmod 1)$ on the space $R / Z$ are conjugated by the mapping $\sigma^{+}: \sum_{m}^{+} / \sim \rightarrow R / Z$ defined by $\Psi\left(x_{1}, x_{2}, x_{3}, \ldots \ldots\right)=\sum_{\mathrm{i}=1}^{\infty} \frac{x_{\mathrm{i}}}{m^{\mathrm{i}}}$.

Proof: We have to establish that (i) $\Psi$ is surjective and (ii) $\Psi o \sigma^{+}=f_{m} o \Psi$. Now since every $m$-adic rational number in $[0,1]$ is the image of two particular sequences in $\sum_{m}^{+}$which are equivalent in $\Sigma_{m}^{+} / \sim$, so every $m$-adic rational has only one and only one pre-image. Also since every non-m-adic rational has only one pre-image in $\sum_{m}^{+} / \sim($ since every non-m-adic rational has unique m-nary expansion), so it immediately follows that $\Psi$ is $1-1$. Therefore, $\Psi$ is a topological conjugacy between $\sigma^{+}$and $f_{m}$.

Theorem 4.6: The map $f_{m}(x)=\operatorname{mx}(\bmod 1)$ on $R / Z$ is Devaney chaotic.

Proof: We have already established that the shift map $\sigma^{+}: \Sigma_{m}^{+} / \sim \rightarrow \sum_{m}^{+} / \sim$ is Devaney chaotic and is topologically conjugate to the map $f_{m}(x)=\operatorname{mx}(\bmod 1)$ on $R / Z$. Since Devaney chaoticity retains under topological conjugacy, so, the map $f_{m}(x)=\operatorname{mx}(\bmod 1)$ on $R / Z$ must be Devaney chaotic.

Theorem 4.7: The forward shift map $\sigma^{+}: \sum_{m}^{+} \rightarrow \sum_{m}^{+}$is exact Devaney chaotic (EDevC).

Proof: Let us first prove that the map $\sigma^{+}: \sum_{m}^{+} \rightarrow \sum_{m}^{+}$is exact. For this, let $\mathrm{U}$ be any non-empty open set in $\sum_{m}^{+}$. We now prove that there is an integer $k \in N$ such that $\sigma^{k}(U)=\sum_{m}^{+}$. Since $U$ is non-empty, so there exists at least one element $x \in U$. Again, since $U$ is open in $\sum_{m}^{+}$, so for $x \in U$, there must exist an open ball $B(x, r)$ such that $B(x, r) \subseteq U$. Then, we 
can choose some $k \in N$ such that $\frac{1}{m^{k}} \leq r$.

If we put $\frac{1}{m^{k}}=r_{1}$, then $r_{1}=\frac{1}{m^{k}} \leq r$ and hence clearly $B\left(x, r_{1}\right) \subseteq B(x, r) \subseteq U$. Then, for every $y \in B\left(x, r_{1}\right)$, we always have that $d(x, y)<r_{1}=\frac{1}{m^{k}}$.

From this, it immediately follows that $x$ and $y$ agree at least up to the $k^{\text {th }}$ term. Also after $k^{\text {th }}$ term, all the sequences in $\sum_{m}^{+}$ may be tails of $y$. That is $B\left(x, r_{1}\right)$ contains all the points whose first $k$ digits agree with $x$ and the tails are all the sequence of $\sum_{m}^{+}$. Hence the $k^{\text {th }}$ iterates of all these points in $B\left(x, r_{1}\right)$ constitute the space $\sum_{m}^{+}$, i.e., $\sigma^{k}\left(B\left(x, r_{1}\right)\right)=\sum_{m}^{+}$.

Also,

$$
\begin{aligned}
& B\left(x, r_{1}\right) \subseteq U \Rightarrow \sigma^{k}\left(B\left(x, r_{1}\right)\right) \subseteq \sigma^{k}(U) \\
& \Rightarrow \sum_{m}^{+} \subseteq \sigma^{k}(U) \\
& \Rightarrow \sum_{m}^{+}=\sigma^{k}(U), \quad\left[\text { Since } \sum_{m}^{+} \supseteq \sigma^{k}(U)\right]
\end{aligned}
$$

Since $U$ is an arbitrary non-empty open set of $\sum_{m}^{+}$, so the result $\sum_{m}^{+}=\sigma^{k}(U)$ is true for every non-empty open set $U$ of $\Sigma_{m}^{+}$. Therefore, $\sigma^{+}$is an exact map on $\Sigma_{m}^{+}$.

So it follows that $\sigma^{+}$is exact Devaney chaotic. As $\sigma^{+}$is exact Devaney chaotic, therefore, it also mixing Devaney chaotic and weak mixing Devaney chaotic.

Here we give an example of a continuous function that is topologically transitive but not chaotic. We also give a suitable example illustrating that all topologically transitive maps are not totally transitive.

Example 4.1: Let $T(x)=1-|2 x-1|$ for $0 \leq x \leq 1$ and let $d_{1}$ be a continuous map from

$\left[-\frac{1}{2}, 1\right]$ to itself defined by $d_{1}(x)=-x$ for $-\frac{1}{2} \leq x \leq 0$ and $d_{1}(x)=T(x)$ for $0 \leq x \leq 1$.

Then $d_{1}$ is chaotic on $\left[-\frac{1}{2}, 1\right]$ but $d_{1}$ is not topologically transitive [7].

Again from the following example, we can see that all topologically transitive maps are not totally transitive.

Example 4.2: Let $J(x)$ be a continuous map from [0,1] onto itself defined by

$$
J(x)=\left\{\begin{array}{cc}
4 x+\frac{1}{5}, & 0 \leq x \leq \frac{1}{5} \\
-4 x+\frac{9}{5}, & \frac{1}{5} \leq x \leq \frac{3}{5} \\
\frac{3}{2}-\frac{3}{2} x, & \frac{3}{5} \leq x \leq 1
\end{array}\right.
$$

We can easily prove that the map $J$ is topologically transitive on $[0,1]$. Here we can see that the subintervals [0, $\left.\frac{3}{5}\right]$ and $\left[\frac{3}{5}, 1\right]$ are invariant under $J^{2}$, so $J^{2}$ is not topologically transitive on $[0,1]$. Hence, $J(x)$ is not totally transitive on $[0,1]$. Therefore, all topologically transitive maps are not totally transitive [10].

\section{Conclusions}

In this paper, we have proved some chaos related properties of the forward shift transformation on $\sum_{m}^{+}$, we have proved some new results by applying the properties of topological conjugacy. To derive most of the results, we have applied the Proximity theorem and metric space properties. We have proved that the shift map is the sensitive dependence on initial conditions in a more explanatory way. We have established that the shift map on $\sum_{m}^{+} / \sim$ is topologically conjugated to the map $f_{m}(x)=m x(\bmod 1)$ on $R / Z$. We have concluded in this paper that $f_{m}(x)=\operatorname{mx}(\bmod 1)$ on $R / Z$ is Devaney chaotic and we see that the shift map $\sigma^{+}: \sum_{m}^{+} \rightarrow \sum_{m}^{+}$is exact Devaney chaotic.

In this article, we have discussed with examples that a continuous function which is topologically transitive but not chaotic in the sense of Du and all topologically transitive maps are not totally transitive. We believe that there are several other aspects of the forward shift map which can be explored. These may be taken up in future works.

\section{Acknowledgement}

We would like to thank Binayak S. Choudhury for reading the manuscript and gave suggestions to improve the manuscripts. 


\section{References}

[1] Douglas, A. L. and Marcus, B. (1995). An Introduction to Symbolic Dynamics and Coding. Cambridge University Press.

[2] Bhaumik, I. and Choudhury, B. S. (2010). A Note on the Genaralized Shift Map. General Mathematics Notes, 1, 154-160.

[3] Bhaumik, I. and Choudhury, B. S. (2010). Some Stronger Chaotic Features of the Genaralized Shift Map. International Journal of Pure and Applied Sciences and Technology, 1(2), 79-86.

[4] Du, B. S. (1998). A dense orbit almost implies sensitivity to initial conditions. Bulletin of the Institute of Mathematics Academia Sinica, 26,85-94.

[5] Biswas H. R. (2017). Investigation of chaoticity of the generalized shift map under a new definition of chaos and compare with shift map, Barisal University Journal Part 1, 4(2), 261-270.

[6] Ju H., Shao H., Choe Y., and Shi Y. (2016). Conditions for maps to be topologically conjugate or semi-conjugate to subshifts of finite type and criteria of chaos. Dynamical Systems, International Journal, 31(4). https: //doi.org/ 10.1080/14689367.2016.1158240.

[7] Biswas H. R. and Monirul M. Islam. (2020). Shift Map and Cantor Set of Logistic Function. IOSR Journal of Mathematics, 16(3), 01-08.

[8] Li, T. Y. and Yorke J. A. (1975). Period three implies chaos. The American Mathematical Monthly, 82(10), 985-992.

[9] Auslander, J. and Yorke, J. A. (1980). Interval maps factors of maps and chaos. Tohoku Mathematical Journal, 32, 177-188.

[10] Devaney, R. L. (1989). An introduction to chaotic dynamical systems. 2nd edition, New York: Addison-Wesley, Redwood City, CA.

[11] Banks, J., Brooks, J., Cairns, G., Davis, G., and Stacey, P. (1992). On Devaney’s definition of chaos. American Mathematical Monthly, 99, 332-334.

[12] Snoha, L. (1992). Dense chaos. Commentationes Mathematicae Universitatis Carolinae, 33, 747-752.

[13] Du, B. S. (2005). On the invariance of Li-Yoke chaos of interval maps. Journal of Difference Equations and Applications, 11, 823-826.

[14] Parry, W. (1966). Symbolic dynamics and transformation of the unit interval. Transactions of the American Mathematical Society, 122(2), 368-378.

[15] Robinson, C. (1999). Dynamical System: Stability, Symbolic Dynamics and Chaos. Second Edition, CRC Press, Boca Raton, FL.

[16] Blanchard, F., Glasner, E., Kolyada, S., and Maass, A. (2000). On Li-Yorke pairs. J. Rei. Ange. Math, 547, 51-68.

[17] Ruette, S. (2003). Chaos for continuous interval maps, www.math.u- psud.fr/ruette/, December.

[18] Shao, H., Shi, Y., and Zhu, Y. (2018). Relationships among some chaotic properties of non-autonomous discrete dynamical systems, Journal of Difference Equations and Applications, https: // doi.org/ 10.1080/10236198.2018.1458101.

[19] Wang, X. and Huang, Y. (2013). Devancy chaos revisited. Topology and its Applications, (160), $455-460$.

[20] Denker, M., C. Grillenberger, C., and Sigmund, K. (1976). Ergodic theory on compact metric spaces. Lecture Notes in Mathematics, 527, Springer-Verlag.

[21] Lu, T., Xhu, P., and Wu, X. (2013). The Retentivity of Chaos under Topological Conjugation. Mathematical Problem in Engineering, Article ID 817831.https://doi.org/10.1155/2013/817831.

[22] Ramos, C. C. (2020). Kinematics in Biology: Symbolic Dynamics Approach, Mathematics. 8 , 339. https://doi.org/10.3390/math8030339.

[23] Daniel, P. B. C., Carlos E. C. S., and Cecilio, P. (2016). A smooth chaotic map with parameterized shape and symmetry. EURSIP Journal on Advances in Signal Processing.

[24] Kitchens, B. P. (1998). Symbolic Dynamics-one sided, Two sided and Countable State Markov Shifts. Universitext, Springer Verlag, Berlin. 